\title{
Philosophiques
}

\section{Géométrie et genèse de l'espace selon Poincaré}

\section{Anastasios Brenner}

Volume 31, numéro 1, printemps 2004

Poincaré et la théorie de la connaissance

URI : https://id.erudit.org/iderudit/008936ar

DOI : https://doi.org/10.7202/008936ar

Aller au sommaire du numéro

Éditeur(s)

Société de philosophie du Québec

ISSN

0316-2923 (imprimé)

1492-1391 (numérique)

Découvrir la revue

Citer cet article

Brenner, A. (2004). Géométrie et genèse de l'espace selon Poincaré. Philosophiques, 31(1), 115-130. https://doi.org/10.7202/008936ar

\section{Résumé de l'article}

L'emploi par Poincaré de la notion de convention au sujet des hypothèses géométriques signale un déplacement par rapport aux problématiques traditionnelles. La découverte des géométries non euclidiennes montre qu'il n’y a pas de cadre spatial unique ; plusieurs systèmes sont possibles. On affirme ainsi l'existence d'un aspect essentiel de la connaissance qui ne dérive pas des faits et ne relève ni de l'inné ni de l'intuition. L'introduction de la notion de convention, dont il s'agit de prendre la mesure, ouvre la voie à une prise en compte des facteurs décisionnels en science. Nous nous proposons, dans cet article, d'analyser l'argumentation de Poincaré en reconstruisant sa position face aux doctrines de Kant et de Mill.
Ce document est protégé par la loi sur le droit d'auteur. L'utilisation des services d'Érudit (y compris la reproduction) est assujettie à sa politique d'utilisation que vous pouvez consulter en ligne.

https://apropos.erudit.org/fr/usagers/politique-dutilisation/ 


\title{
Géométrie et genèse de l'espace selon Poincaré
}

\author{
ANASTASIOS BRENNER \\ Université de Toulouse-Le Mirail \\ brenner@univ-tlse2.fr
}

\begin{abstract}
RÉSUMÉ. - L'emploi par Poincaré de la notion de convention au sujet des hypothèses géométriques signale un déplacement par rapport aux problématiques traditionnelles. La découverte des géométries non euclidiennes montre qu'il n'y a pas de cadre spatial unique; plusieurs systèmes sont possibles. On affirme ainsi l'existence d'un aspect essentiel de la connaissance qui ne dérive pas des faits et ne relève ni de l'inné ni de l'intuition. L'introduction de la notion de convention, dont il s'agit de prendre la mesure, ouvre la voie à une prise en compte des facteurs décisionnels en science. Nous nous proposons, dans cet article, d'analyser l'argumentation de Poincaré en reconstruisant sa position face aux doctrines de Kant et de Mill.
\end{abstract}

\begin{abstract}
By understanding geometrical hypotheses as conventions, Poincaré significantly alters the traditional philosophical discussion of space. The discovery of non-Euclidean geometries proves that there is not a single spatial framework; several systems are possible. Thus, there is an essential feature of knowledge that derives neither from empirical facts nor from intuition or innate understanding. The introduction of the notion of convention, whose significance is at issue here, provides a way of understanding the element of free choice in science. This article aims at analyzing Poincaré's reasoning, by contrasting his viewpoint with that of Kant and Mill.
\end{abstract}

\section{Introduction}

La géométrie constitue sans aucun doute un domaine privilégié chez Poincaré. L'examen critique qu'il propose de cette discipline fournit l'une des origines de sa philosophie. C'est à propos de la science de l'espace que Poincaré introduit la notion de convention si caractéristique de sa doctrine. Une fois sa position déterminée au sujet des axiomes géométriques, il s'engage dans deux directions: d'une part les sciences mathématiques pures, d'autre part les sciences mathématisées de la nature. Si chaque science demande un traitement spécifique, la géométrie sert de référence. C'est encore dans le domaine de la géométrie que la connexion entre les découvertes scientifiques de Poincaré et ses réflexions philosophiques est la plus manifeste: la fécondité des géométries non euclidiennes sur le plan mathématique invite à repenser la notion d'espace.

Poincaré écrit à un moment de transition: la géométrie est en train de subir une révolution. Le statut de la géométrie classique a été bouleversé par la découverte des géométries non euclidiennes. La physique va bientôt changer de langage mathématique. À cet égard, Poincaré nous livre un témoignage sur la série de révolutions dans les domaines mathématique et physique, qui débouche sur la science contemporaine. Il rompt nettement 
avec les réponses traditionnelles. En même temps, la solution qu'il propose rencontre des difficultés que les développements scientifiques et philosophiques ultérieurs révéleront. Mais il se peut que dans cet effort d'évaluation, un aspect de l'œuvre de Poincaré ait été quelque peu négligé: sa démarche, son style ou sa méthode. Kuhn l'a remarqué: le changement de paradigme scientifique correspond à une époque privilégiée pour la réflexion philosophique. Il en va ainsi de la révolution einsteinienne, comme précédemment de la révolution copernicienne. D'où la difficulté du discours épistémologique: lorsqu'un paradigme fait preuve de fécondité, le scientifique peut impunément ignorer la philosophie. Mais tout paradigme finit par s'épuiser. S'ouvre alors une période d'incertitude: le scientifique ne peut plus se passer de philosophie. Poincaré en est un exemple typique. Il reste à savoir comment il conçoit cette réflexion philosophique sur la science. Quelle étape marque-t-il dans le développement de la philosophie des sciences de Comte à nos jours? Afin de répondre à ces questions, nous pouvons convoquer diverses recherches récentes.

Depuis une dizaine d'années, on assiste à un regain d'intérêt pour l'œuvre poincaréenne. Ce retour est dû pour une part à l'évolution de la philosophie des sciences elle-même. Kuhn et ceux qui le suivent ont développé une critique du positivisme logique; ils ont réclamé un rôle pour l'histoire des sciences. L'approche historique associée à la difficulté d'élaborer une nouvelle conception de la science a conduit à revenir sur le développement de la philosophie des sciences, afin de procéder à un véritable examen historique. Le Cercle de Vienne a fait l'objet de travaux nombreux. L'attention s'est naturellement portée sur ses sources, et Poincaré a sa place dans cette histoire, lui que le Manifeste du Cercle de Vienne signale parmi les précurseurs de la conception scientifique du monde. Mais le reflux du positivisme logique en tant que doctrine, les recherches sur les langues naturelles et le développement des sciences cognitives nous incitent à regarder d'un autre œil les réserves de Poincaré à l'égard du logicisme et du formalisme ainsi que sa façon de recourir à la psychologie.

\section{Géométrie et convention}

Dans son article de 1891, "Les géométries non euclidiennes", Poincaré emploie, semble-t-il pour la première fois dans son œuvre, le terme de convention pour qualifier le statut des postulats de la géométrie: "Les axiomes géométriques ne sont [...] ni des jugements synthétiques a priori ni des faits expérimentaux. Ce sont des conventions ${ }^{1}$. " En employant ce terme, Poincaré rejette les deux solutions classiques. Il est clair que l'expression de synthétique a priori renvoie à la conception des mathématiques de

1. Poincaré (1891), p. 773, c'est l'auteur qui souligne; 1902, p. 75 . Le terme de convention ne figurait pas dans les textes antérieurs incorporés au chapitre XII de La Science et l'hypothèse. 
Kant, qui est cité auparavant dans l'article. En refusant que les axiomes géométriques soient des faits expérimentaux, Poincaré s'écarte de la conception énoncée par Mill. Cet auteur a également droit à une mention dans le texte. Nous reviendrons par la suite sur ces deux références.

Mais prenons note du contexte géométrique dans lequel apparaît cette thèse cardinale de Poincaré. Sa grande découverte faite dix ans plus tôt concernait les fonctions fuchsiennes, ou automorphes, et exploitait l'analogie avec les géométries non euclidiennes². La réflexion sur la géométrie en 1891 s'appuie sur ses recherches scientifiques et son expérience de mathématicien. En fait, l'article de 1891 ne fait que prolonger une réflexion déjà entamée. Dans un article antérieur, on trouve déjà ce constat négatif: "On peut se demander [...] ce que sont les hypothèses. Sont-ce des faits expérimentaux, des jugements analytiques ou synthétiques a priori? Nous devons répondre négativement à ces trois questions ${ }^{3}$. " En introduisant le concept de convention, Poincaré cherche à échapper à l'alternative traditionnelle de l'a priori et de l'empirique.

Poincaré introduit, dans son texte, une série de fictions. Ces fictions servent à vulgariser des résultats scientifiques; ce sont aussi des expériences de pensée. Retenons-en une:

Supposons, par exemple, un monde renfermé dans une grande sphère et soumis aux lois suivantes: la température n'y est pas uniforme; elle est maxima au centre, et elle diminue à mesure qu'on s'en éloigne, pour se réduire au zéro absolu quand on atteint la sphère où ce monde est renfermé $[\ldots]$. Je supposerai de plus que, dans ce monde, tous les corps aient le même coefficient de dilatation, de telle façon que la longueur d'une règle quelconque soit proportionnelle à sa température absolue. Je supposerai enfin qu'un objet transporté d'un point à un autre, dont la température est différente, se met immédiatement en équilibre calorifique avec son nouveau milieu ${ }^{4}$.

Nous sommes là en présence d'une géométrie riemannienne à trois dimensions. En un sens, ce monde imaginaire n'est pas si différent du nôtre: les corps qui nous entourent se dilatent sous l'effet de la chaleur, seulement le coefficient de dilatation est variable d'une substance à l'autre, et l'équilibre thermique ne s'établit qu'au bout d'un certain temps. Il apparaît alors que nous avons tendance à dissocier l'espace abstrait de l'espace matérialisé par des corps concrets. Poincaré fait remarquer que «rien dans ces hypothèses n'est contradictoire ou inimaginable». Nous pouvons très bien concevoir de multiples géométries. Celle que nous avons adoptée s'explique en partie par les propriétés prédominantes du monde qui nous environne.

2. Poincaré rappelle les circonstances de cette découverte dans Science et méthode, livre 1, chap. III. Pour une présentation récente, voir J. Mawhin (1998).

3. Poincaré (1887), p. 90.

4. Poincaré (1891), p. 641-642; (1902), p. 89. 
Poincaré cherche ici à présenter au non-spécialiste les géométries non euclidiennes. En effet, dès les années 1820, Lobatchevski bâtit tout un système sans recourir au postulat d'Euclide d'après lequel "par un point on ne peut faire passer qu'une parallèle à une droite donnée ${ }^{5}$ ». Il admettait la possibilité d'une infinité de lignes parallèles. Mais ce n'est qu'au milieu du $\mathrm{XIX}^{\mathrm{e}}$ siècle que la communauté mathématique prit conscience de l'importance de ce résultat. Riemann développa alors un nouveau système, en quelque sorte opposé à celui de Lobatchevski, dans lequel le nombre de parallèles est ramené à zéro. Il restait un long travail consistant à consolider ces découvertes et à les étendre. Poincaré participe à cet effort au cours des années 1880-1890. Cette remise en cause de l'unicité de la géométrie ainsi que l'étrangeté des conséquences des nouvelles géométries donnent lieu à diverses interrogations. Poincaré se met à commenter ses travaux scientifiques, et ses commentaires prennent une tournure de plus en plus philosophique. Ainsi est-il conduit à se pencher sur la question de la nature des axiomes ou hypothèses de la théorie. L'emploi du langage mathématique en physique entraîne encore Poincaré à réfléchir aux rapports entre la géométrie et le monde réel. C'est dans ce contexte qu'il recourt à la notion de convention.

Or on n'a pas assez prêté attention à l'originalité de ce choix. Dupréel se plaignait encore en 1925 du peu de place accordé à la notion de convention dans les dictionnaires philosophiques: "Ce désaccord entre les lexiques et l'usage s'explique sans doute en partie par le fait que c'est assez récemment que les théories "conventionnalistes" ont été imposées à l'attention du public philosophique ${ }^{6}$.» En effet, le terme de convention ne figure dans aucun des dictionnaires philosophiques de langue française en usage aux alentours de 1900, ni dans celui de Franck, ni dans le Vocabulaire de Goblot, ni dans la première édition du vocabulaire de Lalande. On pourrait étendre cette observation aux pays germanophones: le terme de convention ne figure pas non plus dans la première édition du Wörterbuch der philosophischen Begriffe d'Eisler publié en 1899. Ceci est d'autant plus étonnant que nous avons affaire ici à une notion classique: elle remonte aux origines de la philosophie, et son usage est consacré, depuis Platon et Aristote, dans le cadre des discussions au sujet de l'origine du langage et de la nature du régime politique. Seul le Dictionnary of Philosophy and Psychology de Baldwin fait exception, en signalant brièvement l'emploi du terme dans le domaine politique et en renvoyant à Hume. Cette omission ne tardera pas à être réparée. "Convention" apparaît dans la troisième édition du Wörterbuch de 1910, dans laquelle les conceptions de Poincaré sont évoquées. Autour du mot se cristallise tout un débat, et l'entrée correspondante de l'édition suivante est considérablement étoffée. Le néologisme

5. Poincaré (1891), p. 769; (1902), p. 63.

6. Dupréel (1925), p. 283. 
«conventionnalisme» y figure pour désigner la doctrine de Poincaré et de ceux qui le suivent.

On peut certes trouver diverses intuitions antérieures, mais la problématique contemporaine de la convention prend indéniablement son origine chez Poincaré. Schlick fait remarquer que "par un choix convenable, on peut toujours obtenir, sous certaines conditions, une désignation non ambiguë du réel au moyen du concept ». Et il ajoute: "C'est Poincaré qui a introduit le terme " convention" dans ce sens plus étroit dans la philosophie naturelle, et l'une des tâches les plus importantes de cette discipline est d'explorer la nature et la signification des diverses conventions que l'on trouve dans les sciences de la nature ${ }^{7}$.»

Que signifie au juste "convention"? Tout en consignant le terme dans le sens de Poincaré, Lalande fait état de ses réserves dans la seconde édition de son Vocabulaire de 1926: "Les mots convention, conventionnel pris en ce sens ont de graves inconvénients: $1^{\circ}$ ils désignent déjà dans la langue courante, et dans celle de la science, une décision réfléchie prise en commun $[\ldots] ; 2^{\circ}$ même lorsqu'il s'agit de plusieurs individus, il arrive souvent qu'il n'y ait eu aucune entente volontaire entre eux, mais que les décisions concordantes se sont trouvées prises par les uns et les autres parce que, sans être nécessaires, elles étaient raisonnables et naturelles; $3^{\circ}$ enfin ces mots impliquent l'idée, souvent péjorative d'une règle accidentelle, arbitraire, qui n'a point de fondement dans la nature des choses.» Certes, convention se dit de plusieurs manières. À l'instar de certains commentateurs allemands, on pourrait forger une expression spécifique pour désigner chacun de ses sens: fonctionnel, axiomatique, judiciel, normatif et instrumental ${ }^{8}$. Nous préférons énoncer la série de problèmes que résume le terme de convention: le langage de la science, les hypothèses fondamentales, la décision expérimentale, les critères rationnels et la théorie de l'instrument.

\section{Ni Kant ni Mill}

Penchons-nous maintenant sur la référence à Kant que nous avons évoquée plus haut. Poincaré critique, dans la conclusion de son article, la thèse selon laquelle les axiomes de la géométrie sont synthétiques a priori. Dès le début de son texte, il emploie de façon appuyée le vocabulaire kantien. Parmi les axiomes qu'énoncent les traités de géométrie, certains relèvent de l'analyse; Poincaré les écarte du champ de son étude: "Je les regarde comme des jugements analytiques a priori, je ne m'en occuperai pas'. " On

7. Schlick (1918), p. 71.

8. Par exemple Schäfer à la suite de Hübner. Voir Schäfer (1986-1995).

9. Poincaré (1891), p. 769; (1902), p. 63: «Deux quantitiés égales à une même troisième sont égales entre elles." Kant reconnaît de tels jugements en géométrie: "Un petit nombre de principes, supposés par les géomètres, sont, il est vrai, réellement analytiques et 
s'attend donc à ce que Poincaré s'occupe des propositions que Kant qualifiait de synthétiques a priori. Le terme d'analytique revient à plusieurs reprises; il est clair que Poincaré cherche à caractériser, par contraste avec l'analyse, le domaine de la géométrie ${ }^{10}$.

Comment faut-il entendre cette référence à Kant? À première vue, nous avons peu d'éléments pour en juger. Le philosophe allemand n'est cité qu'une seule fois dans l'ouvrage. Même en élargissant notre enquête aux trois autres livres philosophiques de Poincaré, nous ne découvrons qu'une seule série de remarques relatives à Kant dans Science et méthode. On y apprend que Poincaré refuse la réduction des mathématiques à de l'analytique a priori que proposent les logicistes. Il écrit: "Pour M. Couturat, les travaux nouveaux, et en particulier ceux de MM. Russell et Peano, ont définitivement tranché le débat, depuis si longtemps pendant entre Leibnitz et Kant. Ils ont montré qu'il n'y a pas de jugement synthétique a priori ${ }^{11}$.» En revanche, Poincaré ne croit pas le débat tranché. Il laisse entrevoir les limites de sa critique de Kant. Dans le domaine de l'arithmétique et de l'analyse, le concept de synthétique a priori est tout à fait pertinent, et Poincaré s'en sert pour caractériser le raisonnement par récurrence ou principe d'induction complète. L'idée est déjà présente dans la première version du texte tiré d'un article de 1891; elle est exploitée de nouveau dans un article de 1894, "Sur la nature du raisonnement mathématique ", qui formera le premier chapitre de La Science et l'hypothèse. Le livre suit l'ordre classique de classification des sciences. Lorsque Poincaré introduit la notion de convention, il a déjà l'intuition de sa conception de l'arithmétique. Il n'est pas loin d'avoir étendu sa problématique aux hypothèses de la mécanique, dont la nature commode avait été soulignée. La notion de convention vient se glisser, à côté de la tripartition kantienne des jugements, en tant que nouvelle rubrique. Quelle est la signification de cette innovation? Il faut bien reconnaître que Poincaré bouscule la structure de la théorie de Kant; la tâche du philosophe se trouve modifiée: elle consiste à explorer les conventions tacites, les définitions déguisées, et à reconstruire les étapes de la genèse de nos notions fondamentales.

Poincaré ne s'attache pas directement au texte kantien, qu'il ne cite jamais; il se réfère aux idées de Kant, telles qu'elles ont été comprises à son époque. Il convient d'examiner la pensée de Poincaré par rapport aux

reposent sur le principe de contradiction; mais ils ne servent aussi, comme propositions identiques, qu'à l'enchaînement dans la méthode et nullement de principes. »Et il donne les deux exemples suivants: "Le tout est égal à lui-même» et «Le tout est plus grand que sa partie ", (1781-1787), B16.

10. Poincaré (1891), p. 772, 773 ; (1902), p. 71, 73. Pour Kant, la plupart des propositions géométriques, comme celles de l'arithmétique, sont synthétiques a priori. Par exemple: "que la ligne droite soit la plus courte entre deux points, c'est une proposition synthétique » (1781-1787, B16. Cf. B41, A163/B204, 1220/B268, A716/B744).

11. Poincaré (1908), p. 127. Voir p. 129, 152. 
débats qui ont eu cours pendant les deux dernières décennies du XIX ${ }^{e}$ siècle. Dans la première version de son texte, Poincaré signale trois auteurs contemporains: Calinon, Lechalas et Renouvier. En effet, ces auteurs ont publié plusieurs articles dans la Revue philosophique et dans Critique philosophique. Calinon développe sur le plan mathématique ce qu'il appelle la géométrie générale, c'est-à-dire un système géométrique réunissant les présuppositions communes aux géométries d'Euclide, de Lobatchevski et de Riemann ${ }^{12}$. C'est Lechalas qui s'efforce d'en dégager les conséquences philosophiques ${ }^{13}$. Ces nouveaux développements mathématiques nous obligent, à son avis, à rejeter la nécessité traditionnellement attachée à la géométrie euclidienne. Renouvier lui répond, en partisan du "néocriticisme $^{14}$ ». S'il est prêt à retoucher la philosophie kantienne à la lumière des découvertes scientifiques, il veut maintenir l'intuition centrale de Kant. Renouvier refuse à la géométrie générale le même statut que celle d'Euclide. Seule celle-ci est véritable: "D'entre toutes ces classes de propositions composant le contraire du vrai, la classe de l'absurde est celle à laquelle appartient la géométrie imaginaire, parce qu'elle part de la supposition que l'une des lois principales de notre représentation de l'étendue et de la figure n'exprime point une relation réelle ${ }^{15}$. . Couturat, qui vient prendre part au débat, ne se range pas à l'avis de Renouvier. Il ne suffit pas de moderniser le kantisme en évoquant les résultats de la science; nous devons entrer dans la logique interne de la pensée scientifique. Le cas de Poincaré montre que l'on peut donner pleinement signification aux géométries non euclidiennes sans pour autant rejeter le rationalisme. Et Couturat de proposer sa propre lecture: "M. Poincaré donne raison implicitement au criticisme, et apporte à la thèse de l'idéalité de l'espace un argument précieux et, selon nous, décisif ${ }^{16}$.» S'il est vrai que certains arguments de Poincaré pourraient s'interpréter de cette façon, il ne me semble pas que Couturat ait bien saisi le sens de sa conclusion. Il en donne ce commentaire: "Rien n'empêche [...] - que les axiomes géométriques — soient des jugements synthétiques a priori, ce qui ne contredit nullement la théorie de M. Poincaré, à savoir que ce sont des conventions arbitraires, des définitions déguisées. En effet, les postulats sont arbitraires [...]; mais notre auteur reconnaît qu'en fait "notre choix, parmi toutes les conventions possibles, est guidé par des faits expérimentaux"; à moins qu'ils ne s'imposent à l'esprit par une nécessité d'ordre esthétique, pour parler le langage de Kant, parce qu'ils définissent l'espace euclidien, forme a priori de la sensibilité ${ }^{17}$. » Pourtant,

12. Voir Calinon (1889).

13. Voir Lechalas (1890).

14. Tout en s'inspirant de Kant, Renouvier d'efforce d'affiner sa doctrine. Il critique les notions de substance et d'infini actuel et met l'accent sur l'importance de la relation.

15. Renouvier (1891), p. 43.

16. Couturat (1893), p. 74.

17. Couturat (1893), p. 76. 
les éclaircissements de Poincaré montrent que la convention ne saurait être assimilée au synthétique a priori, et il en vient à rejeter expressément l'espace comme forme a priori de la sensibilité ${ }^{18}$.

On pourrait mesurer encore la distance prise par rapport à Kant en étudiant le vocabulaire employé. En localisant le synthétique a priori dans le raisonnement par récurrence, Poincaré opère un déplacement significatif: nous devons distinguer soigneusement, dans un système déductif, les définitions, les axiomes et les démonstrations. Puis, en remontant en deçà de ces éléments du discours mathématique, on dégagera le procédé rationnel très général qui est à l'œuvre. Cette remarque de Le Roy vient éclairer la démarche de Poincaré: "Rien n'existe qui réponde parfaitement à la notion de ce que Kant appelle "pur" , c'est-à-dire qui soit tout à fait indépendant de l'expérience ${ }^{19}$. " Le problème qui sollicite les scientifiques philosophes après Kant est celui des sciences pures et des sciences appliquées. La géométrie n'apparaît plus comme source primaire des mathématiques, mais comme une discipline intermédiaire.

Examinons maintenant la relation que Poincaré entretient avec l'empirisme :

Stuart Mill a prétendu que toute définition contient un axiome, puisqu'en définissant on affirme implicitement l'existence de l'objet défini. C'est aller beaucoup trop loin; il est rare qu'en mathématique on donne une définition sans la faire suivre par la démonstration de l'existence de l'objet défini [...]. Il ne faut pas oublier que le mot existence n'a pas le même sens quand il s'agit d'un être mathématique [...]. Un être mathématique existe, pourvu que sa définition n'implique pas contradiction [...]. Mais si l'observation de Stuart Mill ne saurait s'appliquer à toutes les définitions, elle n'en est pas moins juste pour quelques-unes d'entre elles ${ }^{20}$.

Poincaré fait vraisemblablement allusion au passage suivant du Système de logique de Mill:

Il y a donc une distinction réelle entre les définitions de noms et celles qu'on appelle à tort définitions de choses; mais cette différence consiste en ce que celles-ci énoncent tacitement, en même temps que la signification d'un nom, un point de fait. Cette assertion tacite n'est pas une définition; c'est un postulat $^{21}$.

Mill applique cette théorie de la définition à la géométrie. Les définitions géométriques recouvrent en fait deux propositions: une définition

18. Poincaré (1898), p. 8.

19. Le Roy (1960), p. 10.

20. Poincaré (1891), p. 771-772; (1902), p. 70. Cf. (1908), p. 132.

21. Mill (1843), p. 144; trad. franç., p. 162. Il est à noter que la traduction française, due à Louis Peisse, date de 1866. Elle n'a été réimprimée qu'en 1988, indice de l'oubli dans lequel était tombé Mill entre-temps. 
nominale et une présupposition d'existence. La démonstration repose en fait sur la seconde. Ainsi,

Cette proposition «Un cercle est une figure limitée par une ligne dont tous les points sont à égale distance d'un point intérieur » est appelée la définition du cercle; mais la vraie proposition, dont tant de conséquences découlent et qui est réellement un premier principe en géométrie, est que des figures conformes à cette description existent ${ }^{22}$.

Ce texte écrit en 1843 précède la diffusion des géométries non euclidiennes. On est en présence d'une interprétation empiriste des mathématiques. Bien entendu, Poincaré ne suivra pas entièrement Mill. Il précise qu'existence en mathématiques veut dire non-contradiction; elle ne signifie pas réalité concrète. Mais il retient l'idée d'une dépendance mutuelle des axiomes et des définitions. Ainsi, certaines définitions contiennent des axiomes implicites, et des axiomes peuvent figurer des définitions déguisées.

Toutefois, il faut bien être conscient d'un renversement de la problématique. Poincaré n'accepte d'aucune manière la conception millienne des mathématiques. En effet, il déclare: "De quelque façon qu'on se retourne, il est impossible de découvrir à l'empirisme géométrique un sens raisonnable ${ }^{23}$.» Poincaré met en garde d'autres lecteurs comme Mouret, qui, à l'inverse de Couturat, avait compris son texte comme une défense de l'empirisme ${ }^{24}$. En se servant de la notion de convention, Poincaré marque sa distance à l'égard de l'empirisme de Mill. Car si les définitions contiennent des axiomes, le mathématicien aura pour tâche impérative de les expliciter. Mais une fois tous les axiomes nécessaires formulés, il reste que ces axiomes s'apparentent à des définitions déguisées; en les posant, on choisit une géométrie particulière. Poincaré est donc très proche de ce nominalisme que Mill récuse en évoquant de façon remarquable la notion de convention:

Depuis Aristote, et probablement depuis une époque plus reculée, il a été admis, comme vérité évidente, que la Géométrie est déduite de définitions. Cela put aller assez bien tant que la définition fut considérée comme une proposition expliquant la nature des choses. Mais Hobbes vint, qui rejeta absolument cette conception de la définition et soutint qu'elle ne fait autre chose que déclarer la signification d'un nom. Mais il continua cependant d'affirmer aussi ouvertement que ses prédécesseurs que les archai, Principia, les prémisses originelles des mathématiques et même de toute science étaient les définitions; d'où ce singulier paradoxe, que le système de vérités scientifiques, bien plus, toutes les vérités acquises par le raisonnement, sont

22. Mill (1843), p. 257; trad. franç., p. 294.

23. Poincaré (1902), p. 101.

24. Voir l'échange entre Poincaré et Mouret dans les pages de la Revue générale des sciences; Poincaré (1892). 
déduits des conventions arbitraires - arbitrary conventions - des hommes sur la signification des mots ${ }^{25}$.

Si Mill rejette les entités abstraites, tout comme les nominalistes modernes, il critique ce qu'il appelle le nominalisme de Hobbes.

\section{Crises scientifiques et réflexion épistémologique}

Ainsi Poincaré s'écarte-t-il à la fois de Mill et de Kant. Il reste à comprendre la spécificité de sa position. Poincaré voit dans les développements ultérieurs un apport décisif. Ses interlocuteurs privilégiés sont Riemann, Helmholtz et Sophus Lie; ses travaux présentent des analogies avec ceux de Mach et vont inspirer les analyses du Cercle de Vienne. Poincaré revendique la pertinence d'une étude génétique de la notion d'espace. En parlant des associations d'idées qui ont conduit à l'espace ordinaire, il note: "Sont-ce ces associations [...], qui constituent cette forme a priori dont on nous dit que nous avons l'intuition pure? Alors je ne vois pas pourquoi on la déclarerait rebelle à l'analyse et on me dénierait le droit d'en rechercher l'origine ${ }^{26}$. » Il remonte la pente de l'habitude, il cherche à remettre en cause les associations acquises. Nous ne sommes pas loin de Bergson, et l'on ne s'étonnera pas que Le Roy ait décelé une convergence entre les deux auteurs. Toutefois, Poincaré refuse d'accorder une priorité à la continuité; il émet l'hypothèse que nous construisons le continu à partir d'une discontinuité primordiale ${ }^{27}$.

Prenons le cas de l'espace visuel. Une image des objets extérieurs est projetée sur le fond de la rétine; le tableau comporte seulement deux dimensions. La profondeur de l'espace nous est donnée par autre chose que la vue, à savoir les sensations d'accommodation et de convergence. L'espèce humaine a été amenée à synthétiser et à classer d'une certaine manière l'ensemble de ces sensations, parvenant ainsi aux trois dimensions de l'espace ordinaire. Mais il serait tout à fait possible de maintenir séparées accommodation et convergence, et, de cette manière, de construire une quatrième dimension. Nous pourrions même, en introduisant le toucher, séparer chaque muscle de manière à obtenir une multiplicité de dimensions. Sur le plan théorique, rien ne doit nous arrêter dans l'élaboration de systèmes mathématiques. Ces systèmes ne sont pas seulement légitimes, mais éclairent notre conception ordinaire ${ }^{28}$.

25. Mill (1996), t. 7, p. 144-145; trad. franç., p. 163; c'est moi qui souligne. Il est intéressant de noter, à la suite de H. Pulte, que Carl Gustav Jacobi, peu d'années après, emploiera le terme de convention en un sens positif pour qualifier les hypothèses de la mécanique. Cependant, il n'étend pas cette thèse aux hypothèses de la géométrie et ne développe pas l'idée d'une immunité de la théorie par rapport à l'expérience. Son cours de mécanique analytique, prononcé en 1847-1848, restera inédit jusqu'en 1996. Voir Pulte (2000).

26. Poincaré (1905), p. 96.

27. Voir Poincaré (1905), p. 42.

28. On pourrait noter diverses analogies entre la théorie poincaréenne de l'espace et celle de Mach. Cependant, Poincaré, contrairement à Mach, refuse que les sensations aient un caractère spatial. Voir Poincaré (1898), p. 5. Cf. Mach, (1906), p. 13. 
Dans son exposé de Science et méthode, Poincaré se sert du concept mathématique de coupure. Mais c'est le concept de groupe, employé dans ses écrits techniques, qui est fondamental. Poincaré insiste sur l'importance d'une théorie dynamique. Grâce à la mobilité de notre corps, nous obtenons des indications indispensables sur le monde. Ainsi parvenons-nous à distinguer deux types de changement: externe et interne. Un changement externe peut être compensé par un changement interne. Nous pouvons, par exemple, suivre des yeux un objet en mouvement, retenant l'image de l'objet au centre de l'œil. Les changements s'exercent de différentes manières, et il nous est possible de les associer et de les classer. Mais il faut bien noter ce qu'on fait par là: "La classification n'est pas une donnée brute de l'expérience, parce que la compensation [...] n'est jamais effectivement réalisée [...]. Néanmoins, le fait même que l'esprit ait l'occasion d'accomplir cette opération est dû à l'expérience ${ }^{29}$." L'esprit poursuit pour ainsi dire son mouvement; il outrepasse l'expérience afin de forger des concepts maniables et efficaces.

Le déplacement des corps solides constitue une expérience essentielle dans la formation de la notion d'espace. Nous pouvons composer leurs déplacements. Nous définissons ainsi une opération sur ces déplacements. La compensation d'un changement externe par un changement interne représente alors l'opération inverse. De cette manière est introduit le concept de groupe. Mais en considérant l'ensemble des déplacements comme un groupe, nous dépassons les données de l'expérience; nous transformons des lois expérimentales en lois mathématiques. Et Poincaré de recourir ici à la notion de convention: "Quand l'expérience nous apprend qu'un certain phénomène ne correspond pas $d u$ tout aux lois indiquées, nous l'effaçons de la liste des déplacements. Quand elle nous apprend qu'un certain changement ne leur obéit qu'approximativement, nous considérons ce changement, par une convention artificielle, comme la résultante de deux autres changements composants ${ }^{30}$. On pourrait parler d'une idéalisation. Et Poincaré de tirer du concept de groupe d'autres propriétés de l'espace: son caractère homogène, isotrope et illimité. Enfin, parmi les groupes possibles, on parvient à la géométrie euclidienne en choisissant le groupe le plus simple. Plus que sur le détail de la description, nous souhaitons porter notre attention sur la méthode et ses implications.

La théorie de Poincaré fera l'objet de critiques. Déjà son élève Le Roy reconnaît l'apport de la logique mathématique, tout en marquant la spécificité de la pensée mathématique. En effet, dans La Pensée mathématique pure, issue d'un cours qu'il donne durant les années vingt, Le Roy manifeste une bonne connaissance des développements de la logistique.

29. Poincaré (1898), p. 10. Voir Vuillemin, "L'espace représentatif selon Poincaré », dans Greffe (1996), tome 1, p. 282.

30. Poincaré (1898), p. 11. 
Sachant prendre ses distances avec Poincaré, il donne raison sur de nombreux points à Russell. En particulier, Le Roy signale la convergence des deux penseurs au sujet de la construction de l'espace. En faisant fond sur la théorie des groupes, Poincaré est moins éloigné qu'il ne le pense de Russell, qui s'appuie sur la théorie des ensembles.

De même, Carnap, tout en se réclamant de Poincaré dans ses premiers articles, n'en marque pas moins les limites de son conventionnalisme:

La thèse principale du conventionnalisme [Konventionalismus], établie par Poincaré et continuée par Dingler, énonce que, pour la construction de la physique, on doit poser des conventions [Festsetzungen] qui relèvent de notre libre choix. Il s'ensuit que les constituants du contenu des propositions physiques qui proviennent de ces conventions ne peuvent être confirmés ni réfutés par l'expérience. Le choix de ces conventions ne doit pas être fait d'une manière arbitraire, mais seulement d'après des principes méthodiques définis, parmi lesquels le principe de simplicité, qui détermine en dernière instance la décision ${ }^{31}$.

Il ne s'agit pas seulement de reconnaître la pluralité des options. Certains choix sont plus appropriés que d'autres. On portera l'attention sur les critères de décision.

Poincaré invoquait la simplicité en faveur de la géométrie euclidienne en tant que langage mathématique de la physique. Or la théorie de la relativité et les développements de la physique au cours des années 1910 obligent à reprendre le problème à nouveaux frais. C'est ici que Carnap peut manifester son originalité. Il y a un flottement notoire dans l'appréciation de la simplicité, et il convient de définir plus précisément ce concept. Doit-on préférer la simplicité de la partie mathématique de la théorie physique ou la simplicité de la physique dans son ensemble, y compris les connexions avec la perception? Carnap introduit la fiction d'une physique complètement achevée, ce qui lui permet de laisser les problèmes concrets en suspens et de porter son regard sur la structure de la physique ainsi que sur son rapport avec le système des sciences. Une physique achevée comporterait, outre une pure axiomatique, un dictionnaire permettant de traduire l'un dans l'autre le langage de la perception et le langage de la théorie physique, ainsi qu'une description complète de l'état du monde pour deux instants quelconques du temps. Dès lors, il existe deux manières différentes d'appliquer le principe de simplicité: soit on retient le système axiomatique le plus simple, soit on vise à obtenir la représentation la plus simple des contenus de sensation ${ }^{32}$. Carnap soutient la possibilité de cette seconde voie. Ce qui lui permet de défendre la théorie de la relativité et d'envisager l'unité des sciences. Tout en s'appuyant sur le conventionnalisme, Carnap introduit ici des thèmes qui se retrouvent dans sa Construction logique du monde.

31. Carnap (1923), p. 90; c'est moi qui traduis.

32. Ibid., p. 104. 


\section{Conclusion}

Les écrits philosophiques de Poincaré ont pu dérouter. Il n'est pas facile de distinguer les textes scientifiques des textes philosophiques. Certains articles destinés aux mathématiciens doivent être pris en compte pour parfaire l'exposé de la philosophie poincaréenne de la géométrie. Mais même les articles philosophiques présentent un style original. L'exposé de résultats techniques alterne avec l'examen de problèmes philosophiques. Certes, l'argumentation est impeccable; Poincaré dégage avec précision les conséquences et débusque avec acuité les présupposés. Mais comment intégrer les résultats scientifiques dans le cadre d'une discussion philosophique? Comment adapter le langage philosophique à la méthode scientifique? Il s'agit d'un effort de rapprochement, et Einstein aura lui aussi à dissiper les malentendus qui pourraient surgir entre le philosophe et le scientifique. Dans un texte rétrospectif, il écrit: «Le savant [...] n'a pas le moyen de pousser si loin - que le théoricien de la connaissance - ces tentatives de systématisation en matière de théorie de la connaissance $[. .$.$] ;$ les conditions extérieures que lui imposent les faits d'expérience ne lui permettent pas de se laisser trop restreindre, dans l'édification de son univers conceptuel, par son attachement à une théorie systématique de la connaissance ${ }^{33}$. Parmi les différentes positions qu'il a adoptées au cours de sa vie, celle de Poincaré a pu fournir à un moment donné une réponse pertinente pour la tâche poursuivie.

On voit se mettre en place ici un nouveau rapport entre philosophie et science. L'apparition dans la langue française d'un nouveau vocable, celui d'épistémologie, en est un indice ${ }^{34}$. Ce néologisme permettait aux penseurs du début du $\mathrm{xx}^{\mathrm{e}}$ siècle d'exprimer commodément plusieurs choses: l'autonomie d'une discipline, la distance prise par rapport à cette «philosophie des sciences » inventée par Ampère et illustrée par Comte. En se servant du terme d'épistémologie, on levait des ambiguités; il ne s'agissait plus d'une philosophie émanée de la science ni d'une philosophie scientifique. La nouvelle épistémologie ne comportait pas de condamnation de la métaphysique; elle entrait dans une autre relation avec la philosophie $^{35}$. En posant l'existence de conventions cachées au cœur de la science, Poincaré coupe court aux tentatives traditionnelles de fondation. Une con-

33. Einstein (1989-1993), p. 164.

34. Meyerson (1908), p. XIII. C'est cette occurrence que signalent les grands dictionnaires de la langue française. Mais on trouve des occurrences jusque dans les discussions du Premier Congrès international de philosophie de 1900, qui a dû servir à en propager l'emploi. Voir le résumé du mémoire de Wilbois par Le Roy et la réponse de Russell aux objections, Congrès 1900. Comptes rendus, p. 540-562.

35. Ainsi que l'écrit Le Roy en parlant de la «nouvelle philosophie»: «Il y a en elle quelque chose de vraiment nouveau: une conscience très claire de ce qui l'oppose à la science malgré les rapports étroits qu'elle soutient avec celle-ci. C'est par là notamment qu'elle réalise un progrès sur les doctrines similaires qui l'ont précédée. »(Le Roy, 1901, p. 295.) 
vention est posée: elle ne possède pas de nécessité; elle est simplement motivée. Il est inutile de chercher un enracinement univoque dans l'expérience ou dans la raison. On sort du cadre de la philosophie traditionnelle de la connaissance. Poincaré attire l'attention sur de nouveaux problèmes: la pluralité des langages possibles, les modalités de la décision, la sousdétermination de la théorie par rapport à l'expérience, le rôle des hypothèses en science et le caractère de la pratique expérimentale. Il n'apporte peut-être pas une réponse entièrement satisfaisante à tous ces problèmes. Mais Poincaré définit ici plusieurs thèses majeures de la philosophie des sciences du $\mathrm{Xx}^{\mathrm{e}}$ siècle.

\section{Bibliographie}

Brenner, Anastasios (1996), "La nature des hypothèses physiques selon Poincaré », voir Greffe, 1996, p. 389-395.

- (2002), "The French Connection: Conventionalism and the Vienna Circle», History of philosophy of science (sous la dir. de M. Heidelberger et F. Stadler), Dordrecht, Kluwer, p. 277-286.

- (2003), Les Origines françaises de la philosophie des sciences, Paris, Presses Universitaires de France.

Calinon, A. (1889), "Les espaces géométriques", Revue philosophique, $\mathrm{n}^{\circ}$ 27, p. 588-595.

Carnap, Rudolf (1923), "Über die Aufgabe der Physik und die Anwendung des Grundsatzes der Einfachstheit », Kant-Studien, vol. 28, p. 90-107.

- (1928), Der logische Aufbau der Welt, Hambourg, Meiner, 1961; La Construction logique du monde, trad. franç. de T., Rivain, introduction d'É. Schwartz, Paris, Vrin, 2002.

Congrès international de philosophie (1900-1901), Actes, 3 vol., Paris, Armand Colin; Comptes rendus, Revue de métaphysique et de morale, vol. 8, p. 503698.

Couturat, Louis (1893), "L'année philosophique, par F. Pillon ", Revue de métaphysique et de morale, vol. 1, p. 63-85.

Dupréel, E. (1925), "Convention et raison", Revue de métaphysique et de morale, p. 283-310.

Einstein, Albert (1949), Albert Einstein. Philosopher Scientist (sous la dir. de P. A. Schilpp), La Salle., Open Court, 1982; trad. franç. : Euvres (sous la dir. De F. Balibar), vol. 5, Paris, Seuil, 1991.

Folina, Janet (1994), "Poincaré on Mathematics, Intuition and the Foundations of Science ", Philosophy of Science Association, no 2, p. 217-226.

Giedymin, Jerzy (1982), Science and Convention, Oxford, Pergamon.

Granger, Gilles-Gaston (1996), "Vérité et convention", Philosophia scientice, vol. 1, no 1, p. 3-19.

Greffe, Jean-Louis, G. Heinzmann et K. Lorenz (dir.) (1996), Henri Poincaré, science et philosophie, vol. 1, Paris, Blanchard; vol. 2 et 3, Philosophice scientice, vol. $1, \mathrm{n}^{\circ} 4$.

Heinzmann, Gerhard (1988), «Poincaré's Philosophical Pragmatism and the Problem of Complete Induction ", Fundamenta ScientceI, vol. 9, p. 1-19.

Kant, Immanuel (1781), Critique de la raison pure, dans Euvres philosophiques, (sous la dir. De F. Alquié), trad. franç., 3 vol., Paris, Gallimard, 1980. 
Lechalas, Georges (1890), «La géométrie générale et les jugements synthétiques a priori», Revue philosophique, p. 158-169.

Le Roy, Édouard (1901), "Sur quelques objections adressées à la nouvelle philosophie ", Revue de métaphysique et de morale, vol. 9, p. 292-327, 407-432.

- (1960), La Pensée mathématique pure, Paris, Presses Universitaires de France.

Mach, Ernst (1886), Analyse der Empfindungen; trad. franç.: L'Analyse des sensations

(trad. de F. Eggers et J.-M. Monnoyer), Nîmes, J. Chambon, 1996.

- (1896), Die Principien der Wärmelehre, Leipzig, Barth; trad. ang. : Principles of the Theory of Heat, Dordrecht, Reidel, 1986.

- 1905, Erkenntnis und Irrtum; trad. franç. : La Connaissance et l'erreur (trad. de M. Dufour), Paris, Flammarion, 1908.

- 1906, Space and Geometry, Chicago, Open Court.

Mawhin, Jean (1998), "Henri Poincaré, ou les mathématiques sans œillères ", Revue des questions scientifiques, vol. 169, no 4, p. 337-365.

Meyerson, Émile (1908), Identité et réalité, Paris, Vrin, 1951.

Mill, John Stuart (1843), A System of Logic, dans Collected Works (sous la dir. de J. M. Robson), tomes 7 et 8, Londres, Routledge. 1996; trad franç. de L. Peisse, Bruxelles, Mardaga, 1988.

Poincaré, Henri (1887), "Les hypothèses fondamentales de la géométrie », Bulletin de la Société mathématique de France, vol. 15, p. 203-216.

- (1889-1892), Théorie mathématique de la lumière, 2 vol., Paris, G. Carré.

- (1891), "Les géométries non euclidiennes ", Revue générale des sciences, vol. 2 , p. 769-774.

- (1892), "Sur les géométries non euclidiennes, réponse à M. Mouret ", Revue générale des sciences, vol. 3, 1892, p. 74-75.

— (1898), "Des fondements de la géométrie ", Scientific Opportunism/L’Opportunisme scientifique (textes choisis par L. Rougier, établis par L. Rollet), Bâle, Birkhäuser, 2002.

- (1902), La Science et l'hypothèse, préface de J. Vuillemin, Paris, Flammarion, 1968.

- (1905), La Valeur de la science, préface de J. Vuillemin, Paris, Flammarion, 1970.

- (1908), Science et méthode, Paris, Kimé, 1999.

- (1913), Dernières Pensées, Paris, Flammarion, 1963.

Pulte, Helmut (2000), «Beyond the Edge of Certainty: Reflections on the Rise of Physical Conventionalism ", Philosophia Scientice, vol. 4, n 1, p. 47-68.

Renouvier, Charles (1889), "La philosophie de la règle et du compas ", Critique philosophique, vol. 2, p. 337-349.

- (1891), "La philosophie de la règle et du compas", L'Année philosophique, p. 1-66.

Schäfer, Lothar (1986-1995), "Der Konventionalismus des beginneden 20. Jahrhunderts: Entstchungsbedingungen, Einsichten, Probleme », Handbuch Pragmatischen Denkens (sous la dir de H. Stachowiak), Hambourg, Felix Meitner.

Schlick, Moritz (1918), Allgemeine Erkenntnislehre, Berlin, Springer, 1925; trad. angl: General Theory of Knowledge (trad. de A.E. Blumberg), Vienne, Springer, 1974. 


\section{P Philosophiques / Printemps 2004}

Schmid, Anne-Françoise (2001), Henri Poincaré. Les sciences et la philosophie, Paris, L'Harmattan.

Vuillemin, Jules (1976), "Conventionalisme géométrique et théories des espaces à courbure constante", Science et métaphysique (sous la dir. de S. Docks), Paris, Beauchesne, p. 65-105. 